\title{
Es liegt viel vor uns
}

\section{Daniel Schröpfer}

Dr. med., Medizinischer Direktor Stadtärztlicher Dienst Zürich, Vorstand VLSS

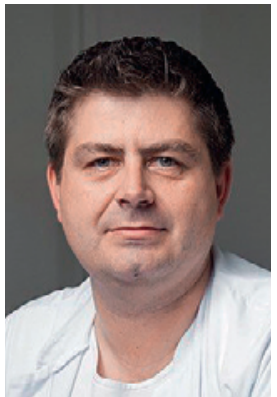

Liebe Leserinnen und Leser

Es freut mich, Sie «zu guter Letzt» begrüssen zu dürfen. Mein Name ist Daniel Schröpfer, ich bin seit Anfang Jahr neues Mitglied in der SÄZ-Redaktion.

Ein paar Worte zu meiner Person: Ich bin Facharzt für Innere Medizin und nach mehreren Leitungsfunktionen seit 2013 bei der Stadt Zürich im ehemaligen «Krankenzimmer für Obdachlose» tätig (heute: Medizinisch-Soziale Ambulatorien im Stadtärztlichen Dienst der Stadt Zürich). Nebenberuflich bin ich seit 2005 berufspolitisch engagiert, und zwar ursprünglich wegen der Umsetzung der 50-Stunden-Woche für Assistenzärzte. Es gab in meinem damaligen Spital niemanden, der einen Dienstplan erstellen konnte oder wollte, der den neuen gesetzlichen Vorgaben entsprach. Als "Youngster» in der kantonalen Ärztegesellschaft lernte ich die Situation der niedergelassenen Ärzte besser kennen. Als Präsident des VSAO durfte ich über mehrere Jahre hinweg die Politik im Sinne der jungen Ärztegeneration aktiv mitgestalten.

Die künftigen Herausforderungen kennen wir alle; ich möchte hier einige, die mir wichtig sind, aufzeigen:

Unsere administrativen Aufgaben nehmen jährlich zu. Werden wir demnächst zum "Verwalter des Patienten", ohne ihn noch selber betreuen zu dürfen? Neue Berufsbilder werden kreiert. Sie sollen uns Ärzte im ambulanten und stationären Bereich im direkten Kontakt mit dem Patienten «entlasten». Dennoch können diese Fachexperten das ärztliche Gespräch, die Anamnese und die klinische Untersuchung nicht ersetzen. Wäre es da nicht sinnvoller, bestehende Berufe weiterzuentwickeln und entsprechende Spezialisten auszubilden, die uns bei der Dokumentation und Abrechnung unterstützen?

Damit zusammen hängt die Frage nach der Attraktivität unseres Berufes. Eins ist klar: Der «Halbgott» in Weiss hat ausgedient. Doch nicht das schwindende Renommee schadet der Attraktivität unseres Berufes, sondern überkommene Arbeitszeitmodelle und die Ökonomisierung.

Arbeitszeiten von mehr als 60 Stunden auf Kaderebene sind unattraktiv und mit dem Privatleben schwer ver- einbar. Natürlich gibt es auch Beispiele, die eine Kombination erlauben. Aber warum werden diese Modelle in Spitälern nicht stärker umgesetzt? Wäre es nicht an der Zeit, nicht mehr von Teilzeitmodellen zu sprechen, sondern von zeitgerechten Anstellungsbedingungen? Je höher man auf Kaderebene steigt, desto reizvoller wird die Aussicht, sollte man meinen. Weit gefehlt: Die Aufgaben sind fast nur noch administrativer Art, und der Gestaltungsspielraum ist gering (je nach Arbeitgeber). Deshalb fehlt den Spitälern hier der Nachwuchs. Die Medizin muss ökonomisiert werden, heisst es. Doch wo liegt der kritische Punkt, an dem sich eine Einsparung in ihr Gegenteil verkehrt? Haben wir ihn nicht schon längst erreicht? Diskussionen mit den Patienten dürfen nach 20 Minuten "abgebrochen» werden, da in der Grundversorgung in der Regel nicht mehr Zeit vorgesehen ist. Patienten werden für ambulante Untersuchungen nach dem stationären Aufenthalt einbestellt, die früher während der Hospitalisation durchgeführt wurden, nur um ein Defizit zu reduzieren. Haben wir hier noch den Patienten im Fokus oder nur die Kosten?

Warum bin ich nun hier? Die SÄZ war und ist eine wichtige Informationsquelle für Ärzte und Ärztinnen in der Schweiz. Für mich ist es zudem eine Zeitschrift, in der breit und offen diskutiert werden kann und in der auch Artikel Platz finden, die nicht dem Mainstream entsprechen. Und die redaktionelle Unabhängigkeit zeugt von ihrem hohen Qualitätsanspruch. Deswegen war ich freudig überrascht, als ich Ende 2019 angefragt wurde, bei der SÄZ mitzuarbeiten.

Es liegt viel vor uns: Die Zeitschriftenformate wandeln sich zusehends, einige Journals erscheinen bereits nur noch elektronisch. Der Anzeigenmarkt im Printbereich bricht mehr und mehr ein. Für mich steht fest: Es wird Veränderungen geben. Und bereits heute möchte ich Sie, liebe Leserinnen und Leser, einladen, sich mit Ihren Rückmeldungen einzubringen. Die SÄZ lebt von Ihrer Mitarbeit, und wir werden Sie künftig auch aktiv(er) darum bitten. Danke für die Zeit, die Sie sich für meinen Artikel genommen haben, und allen, die mich bisher unterstützt haben. 